\title{
DN interaction from the Jülich meson-exchange model
}

\author{
L Tolos $^{*}$, J. Haidenbauer ${ }^{\dagger}$ and G. Krein** \\ *Institut de Ciències de l' Espai (IEEC/CSIC), Campus Universitat Autònoma de Barcelona, Facultat de \\ Ciències, Torre C5, E-08193 Bellaterra (Barcelona), Spain \\ $\dagger$ Institute for Advanced Simulation, Forschungszentrum Jülich, D-52425 Jülich, Germany \\ **Instituto de Física Teórica, Universidade Estadual Paulista, Rua Dr. Bento Teobaldo Ferraz, 271 - 01140-070 \\ São Paulo, SP, Brazil
}

\begin{abstract}
The $D N$ interaction is studied in close analogy to the meson-exchange $\bar{K} N$ potential of the Jülich group using $\mathrm{SU}(4)$ symmetry constraints. The model generates the $\Lambda_{c}$ (2595) resonance dynamically as a $D N$ quasi-bound state. Results for $D N$ scattering lengths and cross sections are presented and compared with predictions based on the Weinberg-Tomozawa term. Some features of the $\Lambda_{c}(2595)$ resonance are also discussed emphasizing the role of the near-by $\pi \Sigma_{c}$ threshold.
\end{abstract}

Keywords: DN interaction, meson-exchange model, $\Lambda_{c}(2595)$ resonance

PACS: 14.40.Lb,13.75.Jz,12.39.Pn

\section{INTRODUCTION}

The study of the interaction of open charm $D$-mesons with nucleons is challenging for several reasons. From the experimental side, reliable models are crucial for guiding planned experiments by the PANDA and CBM experiments of the future FAIR facility at Darmstadt [1]. From the theoretical point of view, the physics motivations are several. Amongst the most exciting ones is the possibility of studying chiral symmetry in matter. Also, studies of $J / \psi$ dissociation in matter [2] require a good knowledge of the interaction of $D$-mesons with ordinary hadrons. Another exciting perspective is the possibility of the formation of $D$-mesic nuclei $[3,4]$ and of exotic nuclear bound states like $J / \psi$ binding to nuclei $[5,6,7]$.

To this end, coupled-channel meson-baryon models in the charm sector have been developed $[8,9,10,11,12$, $13,14]$. In those approaches the strong attraction is provided by vector-meson exchange [10, 14], by the WeinbergTomazawa (WT) term $[9,8,11,12,15]$, or by an extension of the WT interaction to an SU(8) spin-flavor scheme $[13,16]$. All of them obtained dynamically the $\Lambda_{c}(2595)$ resonance. This resonance was reported by the CLEO collaboration [17] and subsequently confirmed by several other experiments [18, 19, 20].

In this paper, the $D N$ interaction is derived in close analogy to the meson-exchange $\bar{K} N$ model of the Jülich group [21]. Results for $D N$ scattering observables are obtained and compared to the outcome of the leading-order SU(4) WT contact term $[11,15]$ and to a SU(8) WT scheme $[13,16]$. We also analyze the $\Lambda_{c}(2595)$ resonance and focus on the consequences of the fact that this resonance coincides practically with the $\pi \Sigma_{c}$ threshold.

\section{DN MODEL IN THE MESON-EXCHANGE FRAMEWORK}

The $D N$ interaction is derived in close analogy to the meson-exchange $\bar{K} N$ model of the Jülich group [21], using as a working hypothesis SU(4) symmetry constraints, and by exploiting also the close connection between the $D N$ and $\bar{D} N$ systems due to G-parity conservation. More specifically, we use the latter constraint to fix the contributions to the direct $D N$ interaction potential while the former one provides the transitions to channels that can couple to the $D N$ system. The main ingredients of the $D N \rightarrow D N$ interaction are provided by vector meson $(\rho, \omega)$ exchange and higher-order box diagrams involving $D^{*} N, D \Delta$, and $D^{*} \Delta$ intermediate states. As far as the coupling to other channels is concerned, we follow here Ref. [21] and take into account only the channels $\pi \Lambda_{c}(2285)$ and $\pi \Sigma_{c}(2455)$. Furthermore, we restrict ourselves to vector-meson exchange and we do not consider any higher-order diagrams in those channels. Pole diagrams due to the $\Lambda_{c}(2285)$ and $\Sigma_{c}(2455)$ intermediate states are, however, consistently included in all channels. We refer the reader to Ref. [22] for details. The resulting interaction potential $\mathscr{V}_{i j}(i, j=$ $D N, \pi \Lambda_{c}, \pi \Sigma_{c}$ ) is then used to calculated the corresponding reaction amplitudes $\mathscr{T}_{i j}$ by solving a coupled-channel 

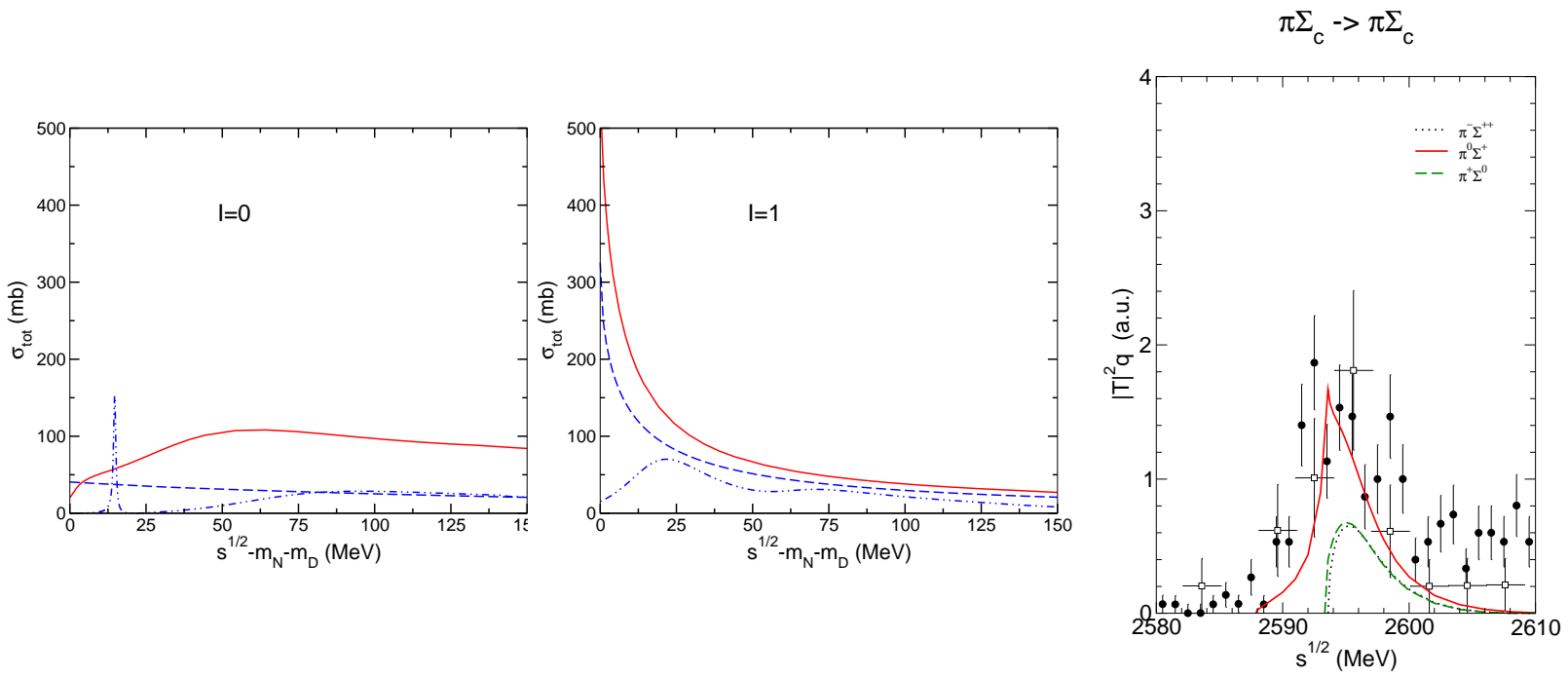

FIGURE 1. Left and middle plots: $D N$ cross sections for the isospin $I=0$ and $I=1$ channels as a function of $\varepsilon=\sqrt{s}-m_{N}-m_{D}$. Results for the Jülich model [22] (solid lines), the SU(4) WT model [11] (dashed lines) and SU(8) WT scheme [13] (dashed-doubledotted lines) are displayed. Right plot: $\pi \Sigma_{c}$ invariant mass spectrum predicted by the Jülich $D N$ meson-exchange model. We show also data for the $\pi^{+} \pi^{-} \Lambda_{c}^{+}$invariant mass distribution taken from [19] (squares) and [20] (circles).

Lippmann-Schwinger-type scattering equation:

$$
\mathscr{T}_{i j}=\mathscr{V}_{i j}+\sum_{k} \mathscr{V}_{i k} \mathscr{G}_{k}^{0} \mathscr{T}_{k j}
$$

from which we calculate the observables in the standard way [23].

\section{DN SCATTERING OBSERVABLES AND THE $\Lambda_{c}(2595)$ RESONANCE}

The SU(4) extension of the Jülich $\bar{K} N$ model to the $D N$ interaction [22] generates narrow states in the $S_{01}$ and $S_{11}$ partial waves which we identify with the experimentally observed $\Lambda_{c}(2595)$ and $\Sigma_{c}(2800)$ resonances, respectively. Not surprisingly, we find an additional pole in the $S_{01}$ partial wave, located close to the other one with a larger width, similarly to the $\bar{K} N$ sector. Our model also generates a further state, namely in the $P_{01}$ partial wave at $2804 \mathrm{MeV}$, i.e. just below the $D N$ threshold. We are tempted to identify this state with the $\Lambda_{c}(2765)$ resonance, whose quantum numbers are not yet established [24].

These results can be compared to previous works on the $D N$ interaction. The SU(4) WT model of Ref. [11] obtains three $S_{01}$ and two $S_{11}$ states up to $2900 \mathrm{MeV}$ [13]. Among them, there is a $S_{11}$ state at $2694 \mathrm{MeV}$ with a width of $\Gamma=153 \mathrm{MeV}$ that strongly couples to the $D N$ channel, with similar effects as the $S_{11}$ resonance of our model. On the other hand, the SU(8) DN WT model [13] predicts even more states in this energy region.

Those resonant states will have a determinant role in some $D N$ scattering observables close to the $D N$ threshold, such as scattering lengths and cross sections. Within the Jülich model we obtain the following scattering lengths for different isospin $(I): a_{I=0}=-0.41+i 0.04 \mathrm{fm}$ and $a_{I=1}=-2.07+i 0.57 \mathrm{fm}$. The large value of the real part of $a_{I=1}$ is due to our $S_{11}$ resonant state. The $S$-wave scattering lengths predicted by our model and by the SU(4) WT approach turn out to be very similar qualitatively for the $I=1$ as well as for the $I=0$ channel. This is in contrast to the SU(8) WT model of [13] which predicts radically different scattering lengths due to the different resonant structure.

The $D N$ cross sections for $I=0$ and $I=1$ are presented in the left and middle plots of Fig. 1 . We show results of the Jülich $D N$ model [22] based on the parameter set that reproduces the positions of the $\Lambda_{c}(2595)$ and $\Sigma_{c}(2800)$ of the Particle Data Group (solid lines). The $D N$ cross sections of the SU(4) WT model of Ref. [11] (dashed lines) and of the SU(8) WT model of Ref. [13] (dash-double-dotted lines) are also displayed. The DN cross sections of the 
SU(4) WT approach of Ref. [11] show a similar behaviour as the one of the Jülich model for the $I=1$ channel. As already discussed above, this model generates likewise poles in the $S_{11}$ partial wave. In case of the $I=0$ channel there are pronounced differences. But this is not surprising because the SU(4) WT model yields only $S$-wave contributions while the results of the Jülich model are dominated by the $P$-wave. Overall larger differences are seen in comparison to the results for the $\mathrm{SU}(8)$ WT model.

Finally, we would like to discuss the $\Lambda(2595)$ resonance and the role of the near-by $\pi \Sigma_{c}$ threshold. The excited charmed baryon $\Lambda_{c}$ (2595) was first observed by the CLEO collaboration [17] and later confirmed by E687 [18] and ARGUS [19], appearing as a pronounced peak in the invariant mass distribution of the $\pi^{+} \pi^{-} \Lambda_{c}^{+}$channel.

In Fig. 1 (right plot) we present results for the $\pi \Sigma_{c}$ invariant mass spectrum in the particle basis for $\pi \Sigma_{c} \rightarrow \pi \Sigma_{c}$ which illustrate the subtle effects of the slightly different thresholds of the $\pi^{+} \Sigma_{c}^{0}, \pi^{0} \Sigma_{c}^{+}$, and $\pi^{-} \Sigma_{c}^{++}$channels on the various invariant mass distributions. Similar invariant mass distributions are obtained for $D N \rightarrow \pi \Sigma_{c}$ channel [22].

The $\pi^{0} \Sigma_{c}^{+} \rightarrow \pi^{0} \Sigma_{c}^{+}$channel resembles very much the measured signal and one can imagine that smearing out our results by the width of the $\Sigma_{c}^{+}$, which is roughly $4 \mathrm{MeV}[20,24]$, would yield a fairly good fit to the data. However, experimentally it was found that the $\Lambda_{c}(2595)$ decays predominantly into the $\pi^{+} \Sigma_{c}^{0}$ and $\pi^{-} \Sigma_{c}^{++}$channels with a branching fraction in the range of $66 \%$ [19] to close to $100 \%$ [20]. Smearing out the corresponding results with the significantly smaller and better known widths of the $\Sigma_{c}^{0}$ and $\Sigma_{c}^{++}$, of just $2 \mathrm{MeV}$ [24], would still leave many of the events found below the nominal $\pi^{+} \Sigma_{c}^{0}$ and $\pi^{-} \Sigma_{c}^{++}$threshold unexplained, especially for the CLEO experiment [20]. Of course, the presence of the $\pi \pi \Lambda_{c}^{+}$channel should be incorporated in our model. But, in any case, it would be important to confirm the new CLEO data by independent measurements of the $\pi \pi \Lambda_{c}^{+}$and $\pi \Sigma_{c}$ mass spectra in the region of the $\Lambda_{c}(2595)$.

\section{ACKNOWLEDGMENTS}

This work is partially supported by the Helmholtz Association through funds provided to the virtual institute "Spin and strong QCD" (VH-VI-231), by the EU Integrated Infrastructure Initiative HadronPhysics2 Project (WP4 QCDnet), by BMBF (06BN9006), by DFG (SFB/TR 16, "Subnuclear Structure of Matter") and FPA2010-16963 from DGI

funds. G.K. acknowledges financial support from CAPES, CNPq and FAPESP (Brazilian agencies). L.T. acknowledges support from the Ramon y Cajal Research Programme (Ministerio de Ciencia e Innovación)

\section{REFERENCES}

1. http://www.gsi.de/fair/

2. T. Matsui, and H. Satz, Phys. Lett. B 178, 416 (1986).

3. K. Tsushima, D. H. Lu, A. W. Thomas, K. Saito, and R. H. Landau, Phys. Rev. C 59, 2824 (1999).

4. C. Garcia-Recio, J. Nieves and L. Tolos, Phys. Lett. B 690, 369 (2010).

5. S. J. Brodsky, I. A. Schmidt, and G. F. de Teramond, Phys. Rev. Lett. 64, 1011 (1990).

6. M.E. Luke, A.V. Manohar, and M.J. Savage, Phys. Lett. B 288, 355 (1992).

7. K. Tsushima, D.H. Lu , G. Krein, and A.W. Thomas, Phys. Rev.C 83, 065208 (2011); G. Krein, A.W. Thomas, and K. Tsushima, Phys. Lett. B 69, 136 (2011)

8. L. Tolos, J. Schaffner-Bielich and A. Mishra, Phys. Rev. C 70, 025203 (2004).

9. M. F. M. Lutz and E. E. Kolomeitsev, Nucl. Phys. A 730, 110 (2004).

10. J. Hofmann and M. F. M. Lutz, Nucl. Phys. A 763, 90 (2005).

11. T. Mizutani and A. Ramos, Phys. Rev. C 74, 065201 (2006).

12. M. F. M. Lutz and C. L. Korpa, Phys. Lett. B 633, 43 (2006).

13. C. Garcia-Recio, V.K. Magas, T. Mizutani, J. Nieves, A. Ramos, L.L. Salcedo, and L. Tolos, Phys. Rev. D 79, 054004 (2009).

14. C. E. Jimenez-Tejero, A. Ramos and I. Vidana, Phys. Rev. C 80, 055206 (2009).

15. L. Tolos, A. Ramos, and T. Mizutani, Phys. Rev. C 77, 015207 (2008).

16. L. Tolos, C. Garcia-Recio and J. Nieves, Phys. Rev. C 80065202

17. K.W. Edwards et al., Phys. Rev. Lett. 74, 3331 (1995).

18. P.L. Frabetti et al., Phys. Lett. B 365, 461 (1996).

19. H. Albrecht et al., Phys. Lett. B 402, 207 (1997).

20. Jiu Zheng, Ph.D. dissertation, University of Florida, 1999.

21. A. Müller-Groeling, K. Holinde, and J. Speth, Nucl. Phys. A 513, 557 (1990).

22. J. Haidenbauer, G. Krein, U. G. Meißner and L. Tolos, Eur. Phys. J. A 47, 18 (2011).

23. R. Büttgen, K. Holinde, A. Müller-Groeling, J. Speth, and P. Wyborny, Nucl. Phys. A 506, 586 (1990).

24. C. Amsler et al. (Particle Data Group), Phys. Lett. B 667, 1 (2008). 